\title{
ESTIMATION OF OPTIMAL WEIGHT VECTORS FOR SPATIAL BROADCAST CHANNELS
}

\author{
Murat Torlak ${ }^{1}$, Guanghan Xu ${ }^{1}$, Brian L. Evans ${ }^{1}$, and Hui Liu ${ }^{2}$ \\ ${ }^{1}$ Department of Electrical and Computer Engineering, The University of Texas, Austin, TX 78712-1084, USA \\ ${ }^{2}$ Department of Electrical Engineering, University of Virginia, Charlottesville, VA 22903-2442 USA *
}

\begin{abstract}
In a multi-transmitter broadcast system, the weight vector for each message signal can provide an additional degree of freedom for signal enhancement and interference suppression by taking advantage of the spatial diversity among the users. The design of optimal weight vectors that maximize the overall channel capacity is an open problem. Under certain power constraints, the channel capacity $R$ is a highly nonlinear function of the $M$-dimensional weight vectors $\left\{\mathbf{w}_{i}\right\}$, where $M$ is the number of transmitters. Hence, a closed-form algebraic solution that maximizes $R$ over $\left\{\mathbf{w}_{i}\right\}$ does not seem to be tractable. In this paper, we decouple the weight vectors in $R$ to simplify the optimization problem to a search for the maxima of a smooth multidimensional function. Based on this decoupling, we derive and evaluate two algorithms for computing weight vectors for the two-user and three-user cases: orthogonal and optimal. We also propose a near-optimum algorithm for the two-user case. The optimal algorithm requires an iterative search.
\end{abstract}

\section{INTRODUCTION}

During the 1990s, the demand for wireless communications and mobile cellular communications has dramatically increased. One way to increase the capacity and improve the performance of existing wireless communications systems is to incorporate the use of spatial diversity. Since Cover's novel work in single-transmitter broadcast channels [4], researchers have been exploring the use of both multitransmitter and multi-receiver systems [1, 3]. In this paper, we focus on multi-transmitter broadcast systems.

A multi-transmitter broadcast channel is a communication channel in which an antenna array system is transmitting to two or more receiving users. In order to optimize performance of the communications system, we must find the optimal weight vector for each message signal that maximizes the overall channel capacity. In this paper, we study

${ }^{*}$ This work was sponsored in part by NSF CAREER Award under Grant MIP-9502695, Office of Naval Research under Grant N00014-95-1-0638, the Joint Services Electronics Program under Contract F49620-95-C-0045, Motorola, Inc., Southwestern Bell Technology Resources, Inc., and Texas Instruments. The United States Government is authorized to reproduce and distribute reprints for governmental purposes notwithstanding any copyright notation hereon. channel capacity assuming only independent decoding since it is more feasible to implement in practical systems than joint decoding.

Recent investigations in wireless communication systems using antenna arrays, called Spatial-Diversity-MultipleAccess (SDMA) systems [2, 5, 6], have attempted to exploit spatial diversity among the users. The design of the optimal weight vectors that maximize the overall channel capacity of the broadcast channels, however, is still an open problem. The primary reason is that under certain power constraints, the channel capacity $R$ is a highly nonlinear function of the $M$-dimensional weight vectors $\left\{\mathbf{w}_{i}\right\}$, where $M$ is the number of transmitters. We presented preliminary results for the two-user case in [7].

In this paper, we decouple the weight vectors in $R$ to simplify the optimization problem to a search for the maxima of a smooth multidimensional function. The multidimensional function has $P(P-1)$ dimensions where $P$ is the number of users. Based on the decoupling of the weight vectors, we derive and evaluate two algorithms for computing weight vectors for the two-user and three-user cases: orthogonal and optimal. We also present a near-optimum algorithm for two-user case. The optimal algorithm requires an iterative search. The orthogonal and near-optimal algorithms are based on closed-form solutions, and are easier to implement than the optimal algorithm, esp. on a programmable digital signal processor.

\section{BACKGROUND}

In [7], we consider maximizing the channel capacity in a two-user broadcast system with multiple transmitters. Now, we consider more general problem formulation. We let $\left\{s_{i}(t)\right\}$ be the message signals. The base station weights each message signal with a weight vector and then transmits the superimposed signal from an array with $M$ elements:

$$
\mathbf{y}(t)=\sum_{i=1}^{d} \beta_{i} \mathbf{w}_{i} s_{1}(t) .
$$

The signals $\left\{s_{i}(t)\right\}$ are assumed to be i.i.d. with Gaussian distribution; $\left\{\mathbf{w}_{i}\right\}$ are normalized weight vectors, i.e., $\left\|\mathbf{w}_{i}\right\|=1, i=1, \ldots, d$; and $\left\{\beta_{i}\right\}$ are the transmitting magnitudes which are subject to certain power constraints. For simplicity, we assume

$$
\beta_{1}^{2}+\cdots+\beta_{d}^{2}=1 .
$$


If independent decoding is employed at the user receivers, then the achievable channel capacity for each user can be expressed as $\log (1+\mathrm{SINR})$, where SINR is the signal-tointerference noise ratio:

$$
R_{i}=\frac{1}{2} \log \left(1+\frac{\alpha_{i}^{2} \beta_{i}^{2} \mathbf{w}_{i}^{H} \mathbf{a}_{i} \mathbf{a}_{i}^{H} \mathbf{w}_{i}}{1+\sum_{j=1, j \neq i}^{d} \alpha_{i}^{2} \beta_{j}^{2} \mathbf{w}_{j}^{H} \mathbf{a}_{i} \mathbf{a}_{i}^{H} \mathbf{w}_{j}}\right)
$$

where $\mathbf{a}_{i}=\left(\begin{array}{llll}a_{i, 1} & a_{i, 2} & \cdots & a_{i, M}\end{array}\right)^{T}$ is a unit vector representing the direction of the spatial signature vector of the $i^{\text {th }}$ user, and $\alpha_{i}$ is the magnitude of the spatial signature vector of the $i^{t h}$ user. The spatial signature vector, a.k.a. the steering vector, of the $i^{t h}$ user represents the propagation pattern of the $i^{t h}$ user, and is equal to $\alpha_{i} \mathbf{a}_{i}$. The noise power has been normalized to unity.

The goal is to select the weight vectors $\left[\begin{array}{lll}\mathbf{w}_{1} & \cdots & \mathbf{w}_{d}\end{array}\right]$ that maximize the total channel capacity $R_{1}+\cdots+R_{d}$. Maximizing the total channel capacity is different than maximizing the signal-to-interference-noise (SINR) ratio for each user, as is seen in (1). Maximizing the SINR, however, is a first-order approximation to the total channel capacity. The first term in the Taylor series for $\log (1+$ SINR $)$ is SINR. To a first-order approximation, (1) is equal to $\mathrm{SINR}_{1}+\cdots+\mathrm{SINR}_{d}$, which is the total SINR.

\section{DECOUPLING WEIGHT VECTORS}

Theorem 1 The optimal weight vectors that maximizes the channel capacity are in signal subspace $\mathbf{A}$ and can be written as linear combinations of spatial signature vectors.

$\underline{\text { Proof: }}$

$$
\mathbf{w}_{i}=\lambda_{a i} \mathbf{A}+\lambda_{b i} \mathbf{B}
$$

where $\mathbf{A}=\left[\begin{array}{lll}\mathbf{a}_{1} & \cdots & \mathbf{a}_{d}\end{array}\right]$ is the spatial signature matrix; $\mathbf{B}=\left[\begin{array}{lll}\mathbf{b}_{d+1} & \cdots & \mathbf{b}_{M}\end{array}\right]$ which denotes the orthogonal subspace of $\mathbf{A}$, i.e., $\mathbf{A} \perp \mathbf{B} ; \lambda_{a i}=\left[\begin{array}{lll}\lambda_{a i, 1} & \cdots & \lambda_{a i, d}\end{array}\right]$ and $\lambda_{b i}=\left[\begin{array}{lll}\lambda_{b i, d+1} & \cdots & \lambda_{b i, M}\end{array}\right]$ Clearly, we have to show that $\left\|\lambda_{b i}\right\|^{2}=0$. As seen from the capacity formulation, $\left\{\lambda_{b i}\right\}$ will not directly contribute to $\kappa_{i j}$ which will be defined in next Section; however, $\left\{\lambda_{b i}\right\}$ will be in the power constraint

$$
\left\|\mathbf{w}_{i}\right\|=\left\|\lambda_{a i}\right\|^{2}+\left\|\lambda_{b i}\right\|^{2}=1 \text {. }
$$

It is obvious that $\left\|\lambda_{b i}\right\|^{2}=0$ should be true not to waste any energy in the orthogonal subspace. A similar theorem is also applicable to the case in which the average SINR is maximized [9].

\section{TWO-USER CASE}

For a given $\left(\beta_{1}, \beta_{2}\right)$ pair and a given $\left(\mathbf{a}_{1}, \mathbf{a}_{2}\right)$ pair, our goal is to find the weight vectors $\mathbf{w}_{1}$ and $\mathbf{w}_{2}$ that optimize the total channel capacity $R=R_{1}+R_{2}$. Denote

$$
\begin{array}{lll}
\kappa_{11}=\mathbf{w}_{1}^{H} \mathbf{a}_{1} \mathbf{a}_{1}^{H} \mathbf{w}_{1} & \kappa_{12}=\mathbf{w}_{2}^{H} \mathbf{a}_{1} \mathbf{a}_{1}^{H} \mathbf{w}_{2} \\
\kappa_{21}=\mathbf{w}_{1}^{H} \mathbf{a}_{2} \mathbf{a}_{2}^{H} \mathbf{w}_{1} & \kappa_{22}=\mathbf{w}_{2}^{H} \mathbf{a}_{2} \mathbf{a}_{2}^{H} \mathbf{w}_{2}
\end{array}
$$

where $\kappa_{i j}=\cos ^{2} L\left(\mathbf{w}_{i}, \mathbf{a}_{j}\right)$ is a measure of the angle between the $i$ th weight vector and the $j$ th spatial signature vector. Thus, each $\kappa_{i j}$ term is confined to $[0,1]$.

It is seen from (1) that for certain $\kappa_{11}$ and $\kappa_{22}, R$ is optimal if the relationship between $\kappa_{21}=\mathbf{w}_{1}^{H} \mathbf{a}_{2} \mathbf{a}_{2}^{H} \mathbf{w}_{1}=$ $\cos ^{2} \angle\left(\mathbf{w}_{1}, \mathbf{a}_{2}\right)$ and $\kappa_{12}=\mathbf{w}_{2}^{H} \mathbf{a}_{1} \mathbf{a}_{1}^{H} \mathbf{w}_{2}=\cos ^{2} \angle\left(\mathbf{w}_{2}, \mathbf{a}_{1}\right)$ are determined. The focus of the problem is to find the maximum values of $\kappa_{11}$ and $\kappa_{22}$ in terms of $\kappa_{21}$ and $\kappa_{12}$ or to find the minimum values of $\kappa_{21}$ and $\kappa_{12}$ in terms of $\kappa_{11}$ and $\kappa_{22}$, respectively. The relationship between can be found by substituting (3) into the first two equations in (4) and $\mathbf{w}_{1}^{H} \mathbf{w}_{1}=1$

$$
\begin{aligned}
\lambda_{11}^{2}+\lambda_{12}^{2}+2 \lambda_{11} \lambda_{12} \sqrt{\zeta} & =1 \\
\lambda_{11}^{2}+\lambda_{12}^{2} \zeta+2 \lambda_{11} \lambda_{12} \sqrt{\zeta} & =\kappa_{11} \\
\lambda_{11}^{2} \zeta+\lambda_{12}^{2} \zeta+2 \lambda_{11} \lambda_{12} \sqrt{\zeta} & =\kappa_{21}
\end{aligned}
$$

where

$$
\zeta=\mathbf{a}_{1}^{H} \mathbf{a}_{2} \mathbf{a}_{2}^{H} \mathbf{a}_{1}=\cos ^{2} \angle\left(\mathbf{a}_{1}, \mathbf{a}_{2}\right) .
$$

so $0 \leq \zeta \leq 1$. Although $\left\{\lambda_{i j}\right\}=r_{\lambda_{i j}} e^{j \theta_{\lambda_{i j}}}$ and $\mathbf{a}_{1}^{H} \mathbf{a}_{2}=$ $\sqrt{\zeta} e^{\theta} \zeta$ are usually complex numbers we can always adjust the phases to cancel out each other without affecting the values of $\kappa_{i j}$ and the norm of weight vectors and spatial signature vectors. Therefore, we can solve the equations in (5) for absolute values of $\lambda_{11}, \lambda_{12}$, and $\kappa_{11}$. And, we found the following relation between $\kappa_{11}$ and $\kappa_{21}$

$$
\kappa_{11}=\left(\sqrt{\zeta \kappa_{21}}+\sqrt{(1-\zeta)\left(1-\kappa_{21}\right)}\right)^{2}
$$

This result was found in [7] by using Langrage multipliers. If we follow the same procedure for $\mathbf{w}_{2}$, we will see that the similar function is true for $\mathbf{w}_{2}$. The channel capacity can be rewritten in terms of $\kappa_{12}$ and $\kappa_{21}$.

The optimization problem is to maximize

$$
\begin{gathered}
\frac{1}{2} \log \left(1+\frac{\gamma_{11}\left(\sqrt{\zeta \kappa_{21}}+\sqrt{(1-\zeta)\left(1-\kappa_{21}\right)}\right)^{2}}{1+\gamma_{12} \kappa_{12}}\right)+ \\
\frac{1}{2} \log \left(1+\frac{\gamma_{22}\left(\sqrt{\zeta \kappa_{12}}+\sqrt{(1-\zeta)\left(1-\kappa_{12}\right)}\right)^{2}}{1+\gamma_{21} \kappa_{21}}\right) .
\end{gathered}
$$

with respect to $\kappa_{12}$ and $\kappa_{21}$ subject to the constraints

$$
\begin{aligned}
& 0 \leq \kappa_{12} \leq \zeta \leq 1 \\
& 0 \leq \kappa_{21} \leq \zeta \leq 1
\end{aligned}
$$

where

$$
\begin{gathered}
\zeta=\mathbf{a}_{1}^{H} \mathbf{a}_{2} \mathbf{a}_{2}^{H} \mathbf{a}_{1}=\cos ^{2} \angle\left(\mathbf{a}_{1}, \mathbf{a}_{2}\right) \\
\gamma_{i j}=\alpha_{i}^{2} \beta_{j}^{2} \text { for } i, j=1,2 .
\end{gathered}
$$

\subsection{Orthogonal Weight Vector Algorithm}

Due to the additional degrees of freedom introduced by multiple transmitters, one can manipulate the complex weight vectors to enhance the desired signal and at the same time, suppress the interference. There exists $\mathbf{w}_{i}$ such that $\mathbf{w}_{i}^{H} \mathbf{a}_{i}=\delta_{i j}$ which can completely eliminate the interference from one to the other. When $\mathbf{w}_{i}^{H} \mathbf{a}_{j}=\delta(i, j)$, the channels are orthogonal and each message can be transmitted without interference. As shown in Figure 1, $\mathbf{w}_{1}$ should be in the direction of $\mathbf{P}_{\mathbf{a}_{2}}^{\perp} \mathbf{a}_{1}$, where $\mathbf{P}_{\mathbf{a}_{2}}^{\perp}$ denotes the projection 
operator to the orthogonal space of $\mathbf{a}_{2}$. Orthogonal weight vectors correspond the following parameter values:

$$
\begin{aligned}
& \kappa_{11}=1-\zeta \quad \kappa_{12}=0 \\
& \kappa_{21}=0 \quad \kappa_{22}=1-\zeta
\end{aligned}
$$

However, this selection is generally not optimal in channel capacity, since the desired signal power at the receiver may be reduced as well.

\subsection{Optimal Weight Vector Algorithm}

To find the optimal weight vectors, we want to maximize the non-linear objective function (8) with respect to variables $\kappa_{11}$ and $\kappa_{22}$ subject to (10). The objective function is continuous and twice differentiable, the constraints are continuous and twice differentiable, and the solution space is convex. As a consequence, the optimization problem has a global maximum. Although a closed-form solution for the global maximum may not exist, numerical methods can be used to search for the global maximum. For example, the global optimum solution will always be found by the iterative Sequential Quadratic Programming method [8]. An alternative iterative search algorithm is proposed below:

1. Choose values for $\kappa_{12}$ and $\kappa_{21}$.

2. For the given $\left(\kappa_{12}, \kappa_{21}\right)$ pair, find the legitimate $\mathbf{w}_{1}$ which maximizes $\cos ^{2} \angle\left(\mathbf{w}_{1}, \mathbf{a}_{1}\right)$ and $\mathbf{w}_{2}$ which maximizes $\cos ^{2} L\left(\mathbf{w}_{2}, \mathbf{a}_{2}\right)$. Evaluate $R$ using (8).

3. Repeat steps 1 and 2 for a finite number of times and identify the optimal pair $\left(\kappa_{12, o p t}, \kappa_{21, o p t}\right)$ which maximize $R$ in (8).

4. The weight vectors that correspond to $\kappa_{12, o p t}$ and $\kappa_{21, \text { opt }}$ in step 2 are the solutions.

Step 3 involves only a two-dimensional searching, which is tractable in general but computational expensive. Next, we derive a near-optimum closed-form solution that is simple to compute.

\subsection{Near-Optimal Weight Vector Algorithm}

After substituting $\kappa_{21}=\cos ^{2}\left(\theta_{2}\right), \kappa_{12}=\cos ^{2}\left(\theta_{1}\right)$, and $\zeta=$ $\cos ^{2}(\phi)$ into (8) and performing logarithmic manipulations, the channel capacity can be rewritten as

$$
\begin{aligned}
\frac{1}{2} \log \left(\mathbf{J}\left(\theta_{1}, \theta_{2}\right)\right)=\frac{1}{2} \log \{ & \left(1+\frac{\gamma_{11} \cos \left(\theta_{2}-\phi\right)^{2}}{1+\gamma_{12} \cos \left(\theta_{1}\right)^{2}}\right) \times \\
& \left.\left(1+\frac{\gamma_{22} \cos \left(\theta_{1}-\phi\right)^{2}}{1+\gamma_{21} \cos \left(\theta_{2}\right)^{2}}\right)\right\} .
\end{aligned}
$$

The maximum total capacity of a two-user broadcasting system depends on two variables, $\theta_{1}$ and $\theta_{2}$. These variables denote the angles between the weight vectors and the spatial signature vectors, as seen in (4). Since the logarithm is a monotonic increasing one-to-one function over the positive numbers, maximizing the total channel capacity in (12) is equivalent to maximizing $\mathbf{J}\left(\theta_{1}, \theta_{2}\right)$. A good initial guess at the optimum value is $\theta_{1}=\phi$ and $\theta_{2}=\phi$, which are the left endpoints of $\kappa_{12}$ and $\kappa_{21}$, respectively, because they bring the cosine terms in the numerators in (12) to 1 . We refined this approximation using perturbation (sensitivity) analysis to find the local optimal value [7]. The near optimum angle between the $i$ th weight vector and the $j$ th spatial signature vector is

$$
\theta_{i}=\phi+2 \cos ^{2}(\phi) \triangle \theta_{i}
$$

where

$$
\triangle \theta_{i}=\frac{\gamma_{j i} \gamma_{j j} \cos (\phi) \sin (\phi)\left(1+\gamma_{i i}+\gamma_{i j} \cos ^{2}(\phi)\right)}{\gamma_{i i}\left(1+\gamma_{j i} \cos ^{2}(\phi)\right)\left(1+\gamma_{j j}+\gamma_{j i} \cos ^{2}(\phi)\right)}
$$

where $i, j=1,2$ and $i \neq j$. Since the perturbation analysis returns an approximation of the local optimum angles, the boundary conditions must be explicitly checked to ensure that the local optimum value is a local maximum. For each angle, two boundary conditions on $k_{i j}$ (i.e., $\theta_{i}=\phi$ and $\theta_{i}=\pi$ ) and one local optimum value (i.e., $\theta_{i}$ in (13)) exist. With two angles, a total of nine angle pairs (three times three) must be substituted into $\mathbf{J}\left(\theta_{1}, \theta_{2}\right)$ and the pair that produces the largest $\mathbf{J}\left(\theta_{1}, \theta_{2}\right)$ value is the local maximum.

Using the closed-form solutions in (13), the near-optimum weight vectors can be found by the following steps:

1. Compute $\zeta=\cos ^{2}(\phi)$ in (6) using the given $\left(\mathbf{a}_{1}, \mathbf{a}_{2}\right)$ spatial signature pair,

2. For $i=1,2$ and $j=1,2$, compute $\gamma_{i j}$ in (10) using the given $\left(\alpha_{i}, \beta_{j}\right)$ values

3. Compute near-optimum $\theta_{1}$ and $\theta_{2}$ angles in (13) and (14).

4. Check boundary conditions and near-optimum angle values by substituting all nine possible angle pairs for $\mathbf{J}\left(\theta_{1}, \theta_{2}\right)$ in 12 and keep the angle pair $\left(\theta_{1}^{\prime}, \theta_{2}^{\prime}\right)$ that gave the largest value of $\mathbf{J}\left(\theta_{1}, \theta_{2}\right)$.

5. The weight vectors which corresponding to $\left(\theta_{1}^{\prime}, \theta_{2}^{\prime}\right)$ are the solutions.

\section{THREE-USER CASE}

For the three-user case, our goal again is to maximize the total channel capacity $R=R_{1}+R_{2}+R_{3}$. As in the two-user case, denote

$$
\kappa_{j i}=\cos ^{2} \angle\left(\mathbf{w}_{i}, \mathbf{a}_{j}\right) .
$$

Now, the focus of the problem is to find the relationship between $\kappa_{i i}$ and $\left\{\kappa_{j i}, j \neq i\right\}$. Applying Theorem 1, we could derive the following relation between $\kappa_{11}$ and $\left(\kappa_{21}, \kappa_{31}\right)$ :

$$
\begin{array}{r}
\kappa_{11}=\left(\sqrt{\zeta_{12} \kappa_{21}}-\sqrt{\zeta_{13} \zeta_{23} \kappa_{21}}+\sqrt{\zeta_{13} \kappa_{31}}-\right. \\
\sqrt{\zeta_{12} \zeta_{23} \kappa_{31}}+\sqrt{\left(1-\zeta_{12}-\zeta_{13}+2 \zeta_{12} \zeta_{13} \zeta_{23}-\zeta_{23}\right)} \times \\
\left.\sqrt{\left(1-\zeta_{23}-\kappa_{21}+2 \zeta_{23} \kappa_{21} \kappa_{31}-\kappa_{31}\right)}\right)^{2} /\left(1-\zeta_{23}\right)^{2}
\end{array}
$$

where $\zeta_{i j}=\cos ^{2} \angle\left(\mathbf{a}_{i}, \mathbf{a}_{j}\right)$. Similar derivations can be carried out to find the relations between $\kappa_{22}$ and $\left(\kappa_{12}, \kappa_{32}\right)$ and between $\kappa_{33}$ and $\left(\kappa_{13}, \kappa_{23}\right)$. Using the relations, channel capacity can be expressed in terms of $\left(\kappa_{21}, \kappa_{31}, \kappa_{12}, \kappa_{32}, \kappa_{13}, \kappa_{23}\right)$. For each $k_{i i}$, the solution amounts to solving a set of linear equations and substituting the result into $\mathbf{w}^{H} \mathbf{w}=1$. The parameter values for orthogonal weight vectors are $\kappa_{i j}=0, i \neq j$ and $\kappa_{11}=\left(1-\zeta_{12}-\zeta_{13}+2 \zeta_{12} \zeta_{13} \zeta_{23}-\zeta_{23}\right) /\left(1-\zeta_{23}\right)$, and $\left(\kappa_{22}, \kappa_{33}\right)$ can be found similar to $\kappa_{11}$. However, as in the 
two-user case this selection is generally not optimal in channel capacity. To find optimal weight vectors for the threeuser case, we have to maximize the channel capacity with respect to variables $\left(\kappa_{21}, \kappa_{31}, \kappa_{12}, \kappa_{32}, \kappa_{13}, \kappa_{23}\right)$. A similar iterative algorithm in Section 4.2. can be used to search for the maximum.

\section{NUMERICAL EXAMPLES}

In this section, numerical examples are given to compare numerical search method and near-optimum closed form solution for the two-user case. We also plot the single antenna capacity and naive time sharing performance. In the numerical example, we consider the case where we have a linear uniform array with 8 antenna elements transmitting to two users. In the example shown in Figure 2, the angle between spatial signature vectors and the gains of spatial signature vectors, respectively, are $\phi=54, \alpha_{1}=1.22$, and $\alpha_{2}=1.333$. Another example is primarily presented to compare the maximum total capacities with the orthogonal weight vectors and the optimal weight vectors with respect to the angle between the spatial signatures. As shown in Figure 3, the difference becomes very significant when the angle between the spatial signature vectors are small.

\section{REFERENCES}

[1] P. Balaban and J. Salz, "Optimum diversity combining and equalization in digital data transmission with applications to cellular mobile radio - Part I: Theoretical Considerations," IEEE Trans. on Communications, vol. 40, no. 5, pp. 885894, May 1992.

[2] P. Balaban and J. Salz, "Optimum diversity combining and equalization in data transmission with application to cellular mobile radio - Part II: Numerical results," IEEE Trans. on Communication, vol. 40, no. 5, pp. 895-907, May 1992.

[3] S. Pillai, Array Signal Processing, Springer Verlag, New York, 1989.

[4] T. Cover and J. Thomas, Elements of Information Theory, John Wiley \& Sons, Inc., New York, NY, 1991.

[5] G. Xu, H. Liu, W. Vogel, H. Lin, S.S. Jeng, and G. Torrence, "Experimental Studies of Space-Division-MultipleAccess Schemes for Spectral Efficient Wireless Communications," Proc. IEEE Int. Conf. on Communications, pp. 800804, May 1994.

[6] B. Suard, G. Xu, H. Liu, and T. Kailath, "Channel capacity of space-division-multiple-access schemes," Proc. IEEE Asilomar Conference on Signals, Systems and Computers, Pacific Grove, CA, pp. 1159-1163, Nov. 1994

[7] M. Torlak, G. Xu, B. Evans, and H. Liu "Optimal weight vectors for broadcast channel" Proc. IEEE Asilomar Conference on Signals, Systems and Computers, Pacific Grove, CA, Nov. 1996.

[8] S. Wright, "Convergence of SQP-like methods for constrained optimization" SIAM Journal on Control and Optimization, vol. 27, no. 1, pp. 13-26, Jan. 1989.

[9] W. Yang and G. Xu, "A new method for designing smart antenna downlink weighting vectors with filter banks", SPIE Conf. on Advanced Signal Proc. Algorithms, Arch., and Implementations, San Diego, CA, Jul. 1997, to be submitted.

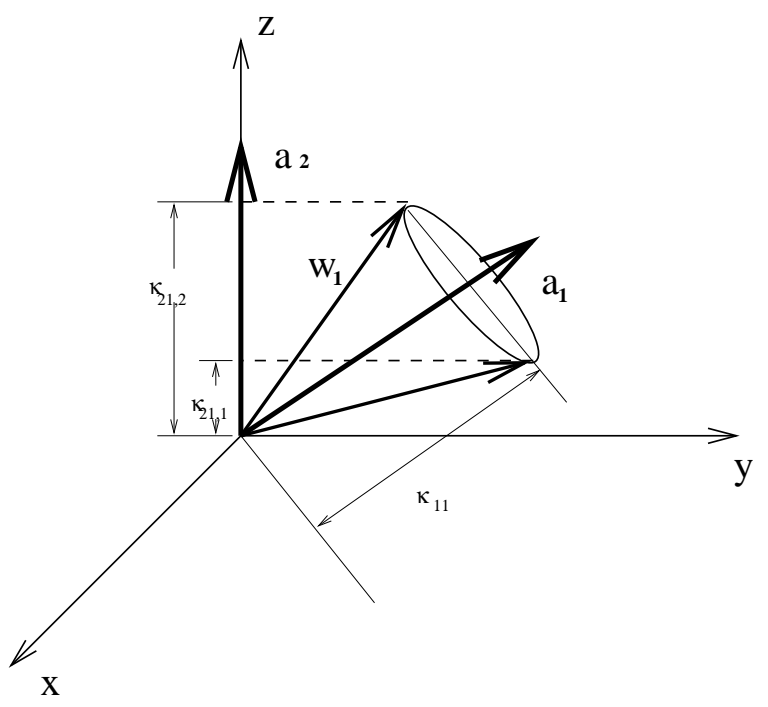

Figure 1. Illustration of search for a optimal weight vector

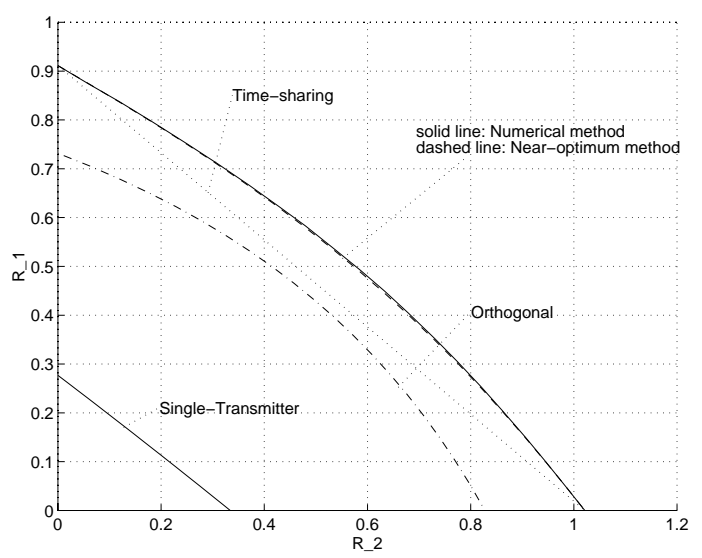

Figure 2. Channel capacities using different weight vectors

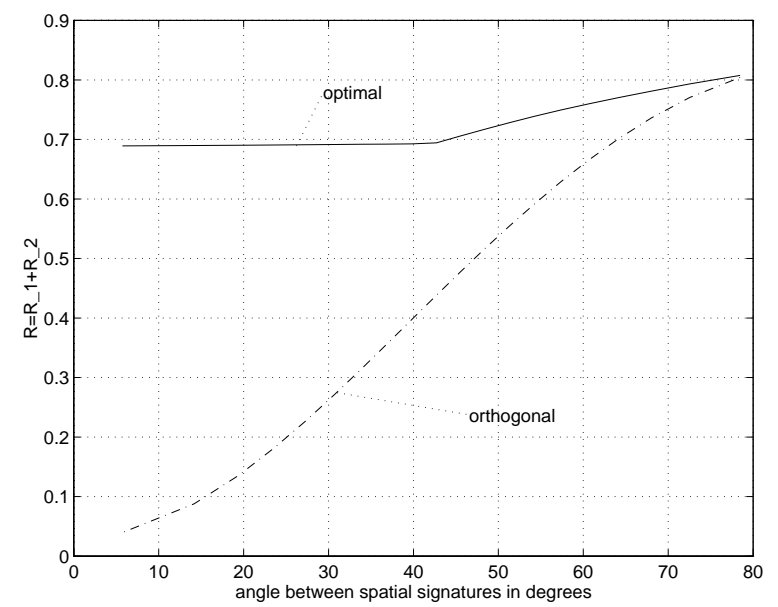

Figure 3. Maximum channel capacities v.s. $\phi$ 